\title{
A Half-Duplex Transmission Scheme for the Gaussian Causal Cognitive Interference Channel
}

\author{
Zhuohua Wu \\ ECE Department, McGill University \\ Montreal, QC, Canada \\ Email: zhuohua.wu@mail.mcgill.ca
}

\author{
Mai Vu \\ ECE Department, Tufts University \\ Medford, MA, USA \\ Email: maivu@ece.tufts.edu
}

\begin{abstract}
The Causal Cognitive Interference Channel (CCIC) models realistic causal cognitive communication between two sender-and-receiver pairs, in which the cognitive sender causally obtains a message from the primary sender and helps forward it to the primary receiver while also sending its own message to the cognitive receiver. We propose a new coding scheme combining the Han-Kobayashi scheme, partial decode-forward relaying and modified dirty-paper coding (DPC) for the Gaussian CCIC in the half-duplex mode. The proposed scheme induces correlation between the transmit signal and the state to allow traditional DPC as well as state forwarding. An achievable rate region with joint decoding is derived. Numerical results show that the rate region for the proposed scheme is better than the Han-Kobayashi scheme and several other existing schemes. We also analyze the maximum rate for the cognitive user while keeping the primary user's rate as interference-free. Results show that, by decodeand-forward relaying, the cognitive user can achieve significant rates while not affecting the primary user's rate even in the half-duplex causal case.
\end{abstract}

\section{INTRODUCTION}

The cognitive interference channel is a four-node channel with two senders and two receivers, in which the second sender knows the message of the first sender so that it can assist in the transmission of the primary sender and its own messages. In recent years, researchers are increasingly interested in the halfduplex mode which is more practical in real systems. The halfduplex causal cognitive interference channel usually consists of two phases. In the first phase, the second user obtains a message from the first sender causally. In the second phase, both senders transmit the messages cognitively.

This channel can model cooperation in practical networks. In the cellular system, consider two base stations with two users near the edge of the cells. User one and user two want to communicate with base station one and base station two, respectively. Suppose that user two is much closer to user one than base station one, then user two can serve as a relay and help user one transmit information. User two can also send its own message to base station two at the same time.

Devroye et al. first propose the concept of an ideal noncausal cognitive radio channel and present an achievable rate region in [1]. They also propose four protocols for the causal case. Time-sharing these 4 protocols can achieve the HanKobayashi rate region but not the decode-forward relaying rate. Chatterjee et al. [2] further study the half-duplex causal cognitive radio channel and propose a better scheme. This scheme can only achieve the rate of decode-forward relaying,
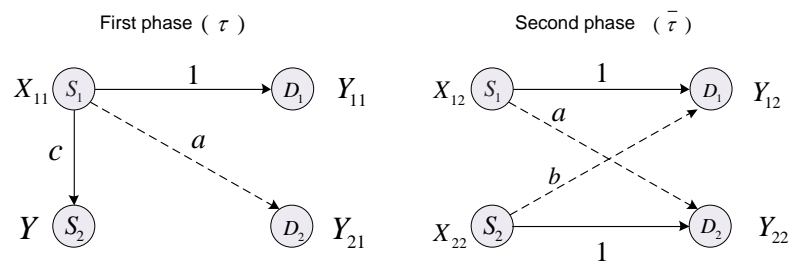

Fig. 1. The half-duplex causal cognitive interference channel model.

which is less than the partial decode-forward rate in the halfduplex mode. We will discuss these two schemes in more detail in Section (III-D). In a related work, Kotagiri and Laneman consider a two-encoder multi access channel (MAC) with one of the encoders having non-causal access to the channel state [3]. They derive an inner bound for the discrete memoryless case and apply a generalized dirty paper coding at the non-causal encoder in the Gaussian case.

In this work, we propose a new coding scheme for the half-duplex cognitive interference channel. The transmission is divided into two phases. In the first phase, the primary sender sends a codeword containing only the cooperative information to the other three nodes. At the end of first phase, the cognitive sender decodes this message part. In the second phase, the primary sender sends a codeword containing all its message parts. The cognitive sender sends a codeword containing its intended messages and the cooperative message decoded from the primary sender in the first phase.

We utilize a modified binning technique at the cognitive sender to not only combat the interference from the primary sender but also help forward part of this primary sender's message. In this scheme, different from traditional dirty paper coding [4], we introduce a correlation between the transmit signal and the state. This correlation helps improve the transmission rate since it contains both functions of binning as in DPC and message forwarding. This idea of the correlation, in fact, has also been used in [3] to allow partial state cancelation (instead of state forwarding as here) in the there considered state-dependent multiple access channel. We derive the rate region for the half-duplex causal cognitive interference channel. We further investigate the optimal time duration for the first transmission phase and the optimal power allocations to achieve the maximum rate for the cognitive sender while keeping the primary sender at the rate without interference. We study the relationships between this maximum rate for 

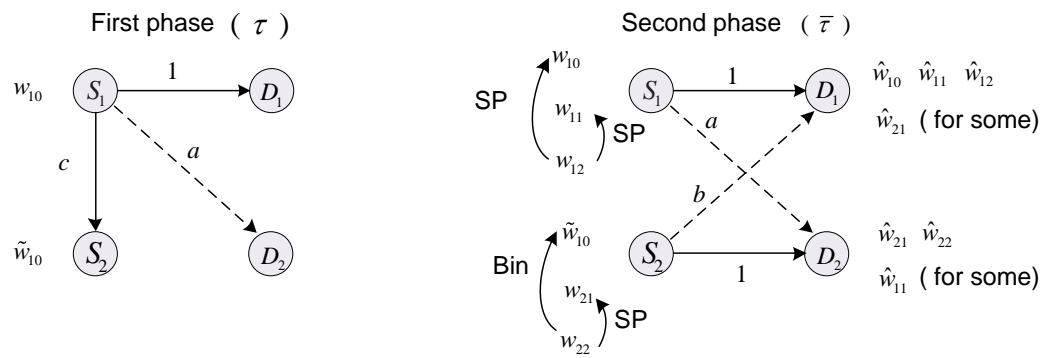

Fig. 2. Coding structure for the Han-Kobayashi partial decode-forward binning scheme $(\mathrm{SP}=$ Superposition, Bin $=\mathrm{Binning})$.

the cognitive sender and three channel gains parameters (two cross channel gains and one channel gain between two transmitters). Simulation results shows that the cognitive user can achieves non-trivial rates while the primary user transmits at its interference-free rate.

\section{Channel Model}

The causal cognitive interference channel consists of four nodes: two senders $S_{1}, S_{2}$ and two receivers $D_{1}, D_{2}$, see Figure 1. $S_{1}$ wants to send a message to $D_{1} . S_{2}$ serves as a causal relay node and helps forward messages from $S_{1}$ to $D_{1}$, while $S_{2}$ also has its own message for $D_{2}$. The transmission in the half-duplex causal cognitive interference channel is divided into two phases. In the first phase, $S_{1}$ transmits while $S_{2}, D_{1}$ and $D_{2}$ listen. In the second phase, both $S_{1}$ and $S_{2}$ transmit and $D_{1}$ and $D_{2}$ listen. This 2-phase transmission allows $S_{2}$ to decode a part of the message from $S_{1}$ in the first phase and then forwards it with its own message in the second phase.

The channel can be represented as

$$
\begin{aligned}
\text { First phase : } \quad Y=c X_{11}+Z \\
Y_{11}=X_{11}+Z_{11} \\
Y_{21}=a X_{11}+Z_{21} . \\
\text { Second phase : } Y_{12}=X_{12}+b X_{22}+Z_{12}, \\
Y_{22}=a X_{12}+X_{22}+Z_{22},
\end{aligned}
$$

where $X_{11}$ is the codeword of $S_{1}$ in the first phase, $X_{12}$ and $X_{22}$ are the codewords of $S_{1}$ and $S_{2}$ in the second phase, respectively. $Y, Y_{11}$ and $Y_{21}$ are the received signals at $S_{2}$, $D_{1}$ and $D_{2}$ in the first phase. $Y_{21}$ and $Y_{22}$ are the received signals at $D_{1}$ and $D_{2}$ in the second phase. $a, b$, and $c$ are the channel gains and the direct links are normalized to 1 as in the standard interference channel [5]. $Z, Z_{11}, Z_{21}, Z_{12}$, and $Z_{22}$ are independent Gaussian noise with unit variance.

The considered half-duplex causal cognitive interference channel is more practical than the cognitive interference channel [1]. The genie-aided assumption in the cognitive interference channel that the cognitive user knows the information of the primary user is impractical in real transmissions. In our proposed scheme, the cognitive user obtains information by decoding, which is more practical in the implementations.

\section{TRANSMISSION SCHEME}

In this section, we introduce a transmission scheme for the half-duplex causal cognitive interference channel based on
Han-Kobayashi, partial decode-forward relaying and Gel'fandPinsker binning techniques. In contrast to traditional binning in dirty paper coding [4], we propose a modified binning technique called "Han-Kobayashi partial decode-forward binning" (or HK-PDF-binning) which introduces a correlation between the transmit signal and the state. The traditional dirty paper coding binning is a special case when this correlation factor is 0 . This modification helps enlarge the rate region as it also allows the partial decode-forward functionality.

\section{A. HK-PDF-binning: A Causal Cognitive Scheme}

In Gaussian channels, signal construction for the partial decode-forward binning scheme is shown in Figure 2. This scheme uses superposition encoding at the first sender, and partial decode-forward decoding and binning at the second sender. The first sender splits its message into three parts $\left(w_{10}, w_{11}, w_{12}\right)$. In the first phase, $S_{1}$ sends the codeword $X_{11}$ containing the message part $w_{10} . S_{2}$ decodes $w_{10}$ from $S_{1}$ at the end of the first phase. Note that neither $D_{1}$ nor $D_{2}$ decodes during this phase. In the second phase, $S_{1}$ sends the codeword $X_{12}$ containing all parts $\left(w_{10}, w_{11}, w_{12}\right)$, in which $w_{11}$ is independent of $w_{10}$, and $w_{12}$ is superimposed on both $w_{10}$ and $w_{11} . S_{2}$ now sends three messages: $w_{21}, w_{22}$ and $w_{10}$. $w_{22}$ is superimposed on $w_{21}$ and conditionally binned against $w_{10}$ (decoded from $S_{1}$ in the first phase with its codeword) given $w_{21}$. We introduce a correlation coefficient $\rho$ between the transmit signal $X_{22}$ and the state $X_{11}$ to help improve the rate region. For decoding, $D_{1}$ uses joint decoding to decode $\left(w_{10}, w_{11}, w_{12}\right)$ using the received signals in both phases. $D_{2}$ decodes $\left(w_{21}, w_{22}\right)$ using the received signal at $D_{2}$ in the second phase.

1) Signal Constructions: Let the relative time duration be $\tau$ and $\bar{\tau}$ for these two phases, respectively, where $\tau+\bar{\tau}=1$. Let $w_{1}$ be the message to be sent by user 1 during a specific block. $S_{1}$ divides it into three parts $\left(w_{10}, w_{11}, w_{12}\right)$ with rate $\left(R_{10}, R_{11}, R_{12}\right)$ and encodes them into $\left(U_{10}, U_{11}, U_{12}\right)$.

In the first phase, the transmitted signals of $S_{1}$ is

$$
X_{11}=\alpha_{1} U_{10}\left(w_{10}\right),
$$

where $U_{10}$ is a Gaussian random variable with unit variance that encodes the message part $w_{10} ; \alpha_{1}^{2}$ is its power allocation. Recall $w_{10}$ is the cooperative message part, which is sent originally by $S_{1}$, decoded by $S_{2}$ and then forwarded to $D_{1}$. $S_{2}$ does not send anything in the first phase but only listens.

In the second phase, the transmit signals of $S_{1}$ is

$$
X_{12}=\alpha_{2} U_{10}\left(w_{10}\right)+\beta_{2} U_{11}\left(w_{11}\right)+\gamma_{2} U_{12}\left(w_{12}\right),
$$


where $U_{11}$ and $U_{12}$, similar to $U_{10}$, are independent Gaussian random variables with unit variance that encode the message part $w_{11}$ and $w_{12}$, respectively. $w_{11}$ is the non-cooperative Han-Kobayashi public message part which is sent by $S_{1}$ and decoded by both $D_{1}$ and $D_{2} ; w_{12}$ is the non-cooperative Han-Kobayashi private message part which is sent by $S_{1}$ and decoded only by $D_{1} ; \alpha_{2}^{2}, \beta_{2}^{2}$ and $\gamma_{2}^{2}$ are the corresponding power allocations for the message parts $w_{10}, w_{11}$ and $w_{12}$. Although the transmit signals in both phases contain message part $w_{10}$, the powers allocated to the message $w_{10}$ are different. Similarly, $S_{2}$ divides its message $w_{2}$ into two parts $\left(w_{21}, w_{22}\right)$ with rate $\left(R_{21}, R_{22}\right)$ and encodes them into $U_{21}$ and $U_{22}$, respectively. $S_{2}$ does not send anything in the first phase. Its transmit signal in the second phase is

$$
X_{22}=\theta U_{21}\left(w_{21}\right)+\mu\left(\rho U_{10}\left(w_{10}\right)+\sqrt{1-\rho^{2}} U_{22}\left(w_{22}\right)\right),
$$

where $w_{10}$ is the cooperative message decoded from $S_{1} ; w_{21}$ is the non-cooperative Han-Kobayashi public message part sent by $S_{2}$ which is decoded by both $D_{1}$ and $D_{2} ; w_{22}$ is the noncooperative Han-Kobayashi private message part decoded only by $D_{2} ; \rho$ is the correlation factor between the transmit signal $X_{22}$ and the state $X_{11} ; \theta^{2}, \mu^{2} \rho^{2}$ and $\mu^{2}\left(1-\rho^{2}\right)$ are the power allocations for the messages $w_{21}, w_{10}$ and $w_{22}$.

The Gelfand-Pinsker binning variable that encodes $w_{22}$ is

$$
\begin{aligned}
V_{22} & =X_{22}+\lambda U_{10}\left(w_{10}\right), \\
& =(\mu \rho+\lambda) U_{10}\left(w_{10}\right)+\theta U_{21}\left(w_{21}\right)+\mu \sqrt{1-\rho^{2}} U_{22}\left(w_{22}\right),
\end{aligned}
$$

where we utilize binning technique to construct $V_{22}$. The codeword for the message part $w_{10}$ is first decoded by sender 2 , and then is treated as the known state to bin with $X_{22} . \lambda$ is the parameter for binning. This $\lambda$ is different from the binning parameter in dirty paper coding [4], in which the codewords for the signal and the binning state are independent. Here we introduce a correlation factor $\rho$ between them. This correlation factor contains the functionalities of both message forwarding and binning. We will give more discussion when deriving the optimal binning parameter $\lambda$.

In the above, $U_{10}, U_{11}, U_{12}, U_{21}$ and $U_{22}$ are independent $\mathcal{N}(0,1)$ random variables. The power constraints at $S_{1}$ and $S_{2}$ are

$$
\begin{aligned}
\tau \alpha_{1}^{2}+\bar{\tau}\left(\alpha_{2}^{2}+\beta_{2}^{2}+\gamma_{2}^{2}\right) & =P_{1}, \\
\bar{\tau}\left(\mu^{2}+\theta^{2}\right) & =P_{2} .
\end{aligned}
$$

2) Decoding: At the end of the first phase, $S_{2}$ uses forward decoding to decode message $w_{10}$ based on the received signals from the first phase. The transmit signal for $S_{1}$ in the first phase is $X_{11}=\alpha_{1} U_{10}\left(w_{10}\right)$, and the received signals at $S_{2}$ is $\mathrm{Y}$ as in (1), from which $S_{2}$ decodes $w_{10} . D_{1}$ and $D_{2}$ do not decode in the first phase.

At the end of the second phase, $D_{1}$ uses sliding window joint decoding to decode $\left(w_{10}, w_{11}, w_{12}\right)$ based on signals received in both the first and second phases (see $Y_{11}$ and $Y_{12}$ in (1) and (2)). $D_{1}$ also decodes $w_{21}$, one part of the message for source 2, but does not care if this decoding is correct.

Similarly, $D_{2}$ uses sliding window joint decoding to decode $\left(w_{21}, w_{22}\right)$ based on the received signals in both phases (see
$Y_{21}$ and $Y_{22}$ in (1) and (2)). $D_{2}$ also decodes one part message $w_{11}$ belonging to source 1 , but does not care if this decoding is successful.

\section{B. Achievable Rate Region}

Theorem 1. The achievable rate region for the half-duplex causal cognitive interference channel using the proposed scheme consists of the convex hull of all rate pairs satisfying

$$
\begin{aligned}
R_{1} & \leq \min \left\{I_{1}+I_{4}, I_{5}\right\} \\
R_{2} & \leq I_{11} \\
R_{1}+R_{2} & \leq \min \left\{I_{1}+I_{6}, I_{7}\right\}+I_{12}, \\
R_{1}+R_{2} & \leq \min \left\{I_{1}+I_{2}, I_{3}\right\}+I_{13}, \\
R_{1}+R_{2} & \leq \min \left\{I_{1}+I_{8}, I_{9}\right\}+I_{10}, \\
2 R_{1}+R_{2} & \leq \min \left\{I_{1}+I_{2}, I_{3}\right\}+\min \left\{I_{1}+I_{8}, I_{9}\right\}+I_{12}, \\
R_{1}+2 R_{2} & \leq \min \left\{I_{1}+I_{6}, I_{7}\right\}+I_{10}+I_{13},
\end{aligned}
$$

where

$$
\begin{aligned}
& I_{1}=\tau C\left(c^{2} \alpha_{1}^{2}\right) \text {, } \\
& I_{2}=\bar{\tau} C\left(\frac{\gamma_{2}^{2}}{b^{2} \mu^{2}\left(1-\rho^{2}\right)+1}\right), \\
& I_{3}=\tau C\left(\alpha_{1}^{2}\right)+\bar{\tau} C\left(\frac{\left(\alpha_{2}+b \mu \rho\right)^{2}+\gamma_{2}^{2}}{b^{2} \mu^{2}\left(1-\rho^{2}\right)+1}\right), \\
& I_{4}=\bar{\tau} C\left(\frac{\beta_{2}^{2}+\gamma_{2}^{2}}{b^{2} \mu^{2}\left(1-\rho^{2}\right)+1}\right), \\
& I_{5}=\tau C\left(\alpha_{1}^{2}\right)+\bar{\tau} C\left(\frac{\left(\alpha_{2}+b \mu \rho\right)^{2}+\beta_{2}^{2}+\gamma_{2}^{2}}{b^{2} \mu^{2}\left(1-\rho^{2}\right)+1}\right), \\
& I_{6}=\bar{\tau} C\left(\frac{\gamma_{2}^{2}+b^{2} \theta^{2}}{b^{2} \mu^{2}\left(1-\rho^{2}\right)+1}\right) \text {, } \\
& I_{7}=\tau C\left(\alpha_{1}^{2}\right)+\bar{\tau} C\left(\frac{\left(\alpha_{2}+b \mu \rho\right)^{2}+\gamma_{2}^{2}+b^{2} \theta^{2}}{b^{2} \mu^{2}\left(1-\rho^{2}\right)+1}\right), \\
& I_{8}=\bar{\tau} C\left(\frac{\beta_{2}^{2}+\gamma_{2}^{2}+b^{2} \theta^{2}}{b^{2} \mu^{2}\left(1-\rho^{2}\right)+1}\right), \\
& I_{9}=\tau C\left(\alpha_{1}^{2}\right)+\bar{\tau} C\left(\frac{\left(\alpha_{2}+b \mu \rho\right)^{2}+\beta_{2}^{2}+\gamma_{2}^{2}+b^{2} \theta^{2}}{b^{2} \mu^{2}\left(1-\rho^{2}\right)+1}\right), \\
& I_{10}=\bar{\tau} C\left(\frac{\mu^{2}\left(1-\rho^{2}\right)}{a^{2} \gamma_{2}^{2}+1}\right), \\
& I_{11}=I_{10}+\bar{\tau} C\left(\frac{\theta^{2}}{\left(a \alpha_{2}+\mu \rho\right)^{2}+a^{2} \gamma_{2}^{2}+\mu^{2}\left(1-\rho^{2}\right)+1}\right), \\
& I_{12}=I_{10}+\bar{\tau} C\left(\frac{a^{2} \beta_{2}^{2}}{\left(a \alpha_{2}+\mu \rho\right)^{2}+a^{2} \gamma_{2}^{2}+\mu^{2}\left(1-\rho^{2}\right)+1}\right), \\
& I_{13}=I_{10}+\bar{\tau} C\left(\frac{a^{2} \beta_{2}^{2}+\theta^{2}}{\left(a \alpha_{2}+\mu \rho\right)^{2}+a^{2} \gamma_{2}^{2}+\mu^{2}\left(1-\rho^{2}\right)+1}\right),
\end{aligned}
$$

where $C(x)=0.5 \log _{2}(1+x) ; \tau \in[0,1]$ and $\rho \in[-1,1]$.

Sketch of proof: According to the encoding and decoding procedures in Section III-A, we can get the rate region in Theorem 1. $I_{1}$ comes from the decoding of $w_{10}$ at $S_{2} . I_{2}-I_{9}$ are due to the joint decoding of $\left(w_{10}, w_{11}, w_{12}\right)$ for some $w_{21}$ at $D_{1} \cdot I_{10}-I_{13}$ are due to the joint decoding of $\left(w_{21}, w_{22}\right)$ for some $w_{11}$ at $D_{2}$. The rate analysis follows standard techniques and is omitted here because of the lack of space. Details can be found in [6] for interested readers. 
1) The maximum $R_{1}$ and $R_{2}$ : The maximum rate for $S_{1}$ is achieved by setting $\beta_{2}=\theta=0, \rho= \pm 1$ and $\mu=\rho \sqrt{\frac{P_{2}}{\bar{\tau}}}$ as

$$
\begin{aligned}
R_{1}^{\max }= & \max _{\tau \alpha_{1}^{2}+\bar{\tau}\left(\alpha_{2}^{2}+\gamma_{2}^{2}\right) \leq P_{1}} \min \left\{\tau C\left(c^{2} \alpha_{1}^{2}\right)+\bar{\tau} C\left(\gamma_{2}^{2}\right),\right. \\
& \left.\tau C\left(\alpha_{1}^{2}\right)+\bar{\tau} C\left(\left(\alpha_{2}+b \sqrt{P_{2}}\right)^{2}+\gamma_{2}^{2}\right)\right\} .
\end{aligned}
$$

A solution for this optimization problem is available in [7]. Note that in the half-duplex mode, partial decode-forward achieves a strictly higher rate than full decode-forward for the Gaussian channel.

The maximum rate for $S_{2}$ is achieved by setting $\tau=0$, $\rho=0, \alpha_{1}=\alpha_{2}=\beta_{2}=\gamma_{2}=\theta=0$, and $\mu=\sqrt{P_{2}}$ as

$$
R_{2}^{\max }=C\left(P_{2}\right) .
$$

2) Special case: PDF-Binning Scheme: In this scheme, $S_{1}$ only splits its message into 2 parts: $w_{10}$ and $w_{12}\left(w_{11}=\emptyset\right)$ and $S_{2}$ does not split its message. $w_{10}$ is first decoded by $S_{2}$, and then forwarded to $D_{1} \cdot w_{12}$ is decoded only by $D_{1} . S_{2}$ bins codewords for $w_{2}$ against codewords for $w_{10}$.

Set $\beta_{2}=\theta=0$ in (8), we get the PDF-Binning rate region

$$
\begin{aligned}
& R_{1} \leq \tau C\left(c^{2} \alpha_{1}^{2}\right)+\bar{\tau} C\left(\frac{\gamma_{2}^{2}}{b^{2} \mu^{2}\left(1-\rho^{2}\right)+1}\right), \\
& R_{1} \leq \tau C\left(\alpha_{1}^{2}\right)+\bar{\tau} C\left(\frac{\left(\alpha_{2}+b \mu \rho\right)^{2}+\gamma_{2}^{2}}{b^{2} \mu^{2}\left(1-\rho^{2}\right)+1}\right), \\
& R_{2} \leq \bar{\tau} C\left(\frac{\mu^{2}\left(1-\rho^{2}\right)}{a^{2} \gamma_{2}^{2}+1}\right),
\end{aligned}
$$

where $\alpha_{1}, \alpha_{2}, \gamma_{2}$ and $\mu$ satisfy the power constraints

$$
\tau \alpha_{1}^{2}+\bar{\tau}\left(\alpha_{2}^{2}+\gamma_{2}^{2}\right) \leq P_{1} ; \quad \bar{\tau} \mu^{2} \leq P_{2} .
$$

Although this scheme cannot achieve the same maximum rate for $S_{1}$ as (9), it can achieve the maximum rate of (10) for $S_{2}$. More important, since $S_{1}$ and $S_{2}$ reduces the complexity of their message splitting, the encoding and decoding procedures become much easier in practical implementations.

\section{Analysis of the binning parameter}

1) Optimal binning parameter: In this section, we will derive the optimal binning parameters for the proposed halfduplex scheme. The intuition for this optimal binning parameter comes from the binning variables in DPC [4]. However, unlike the traditional binning procedure in DPC, we introduce correlation between the state $\left(U_{10}\right)$ and the transmitted codeword $\left(X_{22}\right)$, as can be seen in (5) and (6). This optimal binning parameter is obtained by maximizing the rate region in (8). The terms dependent on this binning parameter are $I_{10}-I_{13}$. One interesting point is that we only need to optimize the term for $I_{10}$ in order to optimize the rate region. This is because we can decompose the terms $I_{11}-I_{13}$ into $I_{10}$ and another constant term that is independent of the binning parameter.

Corollary 1. The optimal parameter $\lambda$ for the proposed scheme is

$$
\lambda^{*}=\frac{a \alpha_{2} \mu^{2}\left(1-\rho^{2}\right)-\mu \rho\left(a^{2} \gamma_{2}^{2}+1\right)}{a^{2} \gamma_{2}^{2}+\mu^{2}\left(1-\rho^{2}\right)+1} .
$$

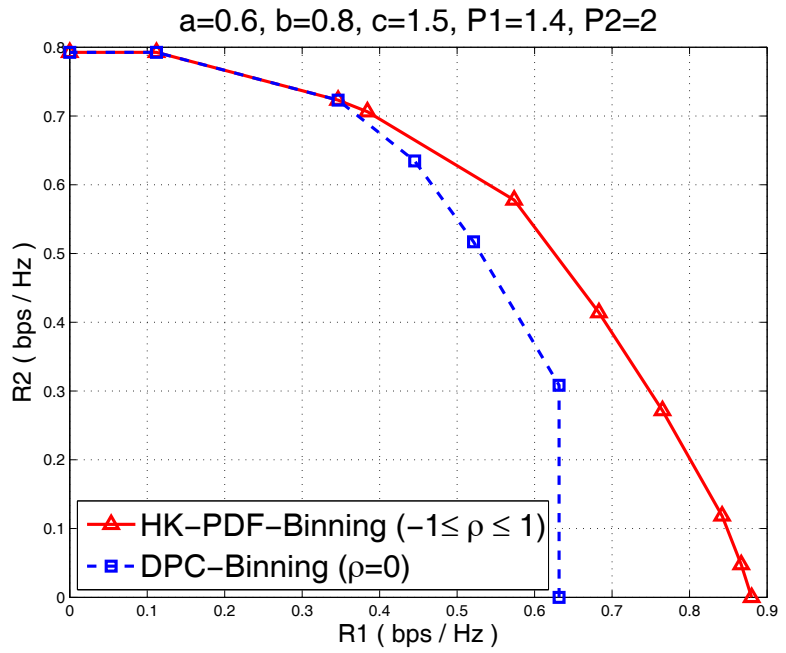

Fig. 3. Effect of $\rho$.

Proof: The optimal $\lambda$ is obtained by maximizing the term $I_{10}$ in Theorem 1. The detailed calculation for optimal $\lambda$ can be seen in [6].

2) The role of the binning parameter: The signal composition at $S_{2}$, which consists of three parts, is shown in (5). $U_{21}$ contains the Han-Kobayashi public message part at $S_{2}$. $U_{10}$ is the forwarding message part decoded from $S_{1}$ and is also the state known to $S_{2} . U_{22}$ is a part of the binning signal in (6). Note that in this binning signal, the transmit signal $X_{22}$ is not independent of the state $U_{10}$ as in traditional DPC. This is because the state is also forwarded by the transmit signal. Therefore the optimal binning parameter functions as both message forwarding and traditional DPC binning. The correlation parameter $\rho$ adjusts the portion of message forwarding and DPC binning at $S_{2}$. For example, if we set $\rho= \pm 1, X_{22}$ will only encode $w_{10}$ and $w_{21}$ without any actual binning, hence realize the function of message forwarding. If we set $\rho=0$, the proposed scheme becomes dirty paper coding without any message forwarding. For $0<|\rho|<1$, it has both the functions of binning and message forwarding. When $|\rho|$ is approaching to $1, S_{2}$ allocates more power for message forwarding; when $|\rho|$ is approaching to $0, S_{2}$ acts more as dirty paper coding. Thus, the proposed scheme generalizes dirty paper coding.

The effect of $\rho$ is shown in Figure 3. The dashed line represents the rate region using only DPC-binning $(\rho=0)$, while the solid line represents the region for the proposed scheme when we adapt $\rho \in[-1,1]$. Figure 3 illustrates that the correlation factor $\rho$ can enlarge the rate region.

\section{Numerical Results}

We compare the proposed schemes with the Han-Kobayashi and other two known half-duplex coding schemes [1], [2] in Figure 4. Devroye, Mitran and Tarokh propose four halfduplex protocols in [1]. One protocol is the Han-Kobayashi scheme for the interference channel, and the other three are 2phase protocols. All these 3 protocols decode the cooperative message at the end of both phases instead of only at the end of the second phase jointly, hence they are suboptimal and do not 


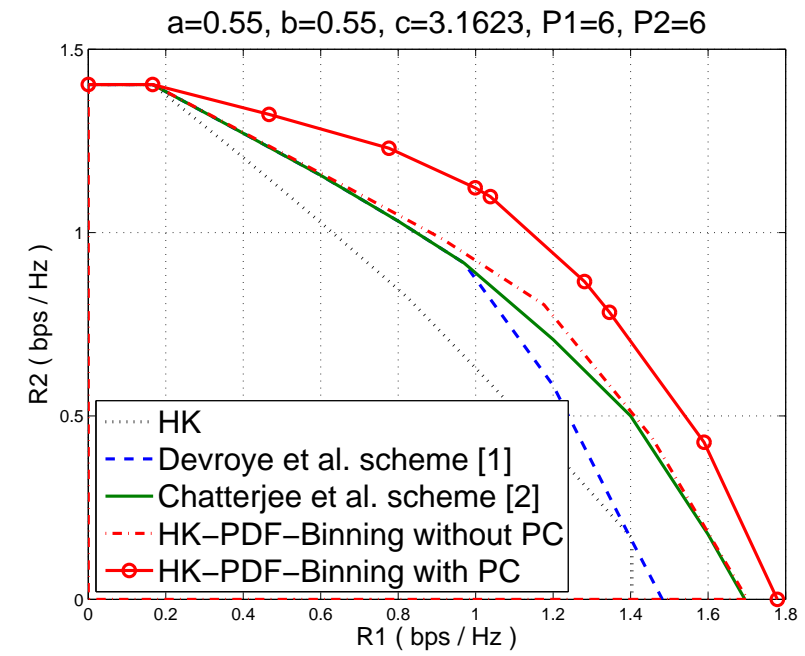

Fig. 4. Comparison of the combined scheme with existing schemes. $(\mathrm{PC}=$ power control)

include the partial decode-forward relaying rate. Chatterjee, Wong and Oyman [2] propose an achievable rate region for the half-duplex CCIC based on rate-splitting, block Markov encoding, Gelfand-Pinsker binning and backward decoding. But their scheme only covers half-duplex full decode-forward relaying (when there is no binning) instead of partial decodeforward relaying and hence achieves a maximum rate for $R_{1}$ smaller than (9). Since both these existing schemes have constant power in both phases, we plot the achievable rate region of our proposed scheme in 2 cases: with power control as in (7) and without (i.e. constant power). In Figure 4, we can see that the proposed scheme is strictly better than these three existing schemes either with or without power control.

\section{MAXimum Non-InTERfERence Rate for the Cognitive User}

In this section, we analyze the proposed scheme to find the maximum $R_{2}$, the optimal parameter $\tau$ and power allocations, under the constraint $R_{1}=C\left(P_{1}\right)$. This problem has several practical considerations. First, we are interested from the practical point of view in the maximum rate that the cognitive user can transmit while the primary user still transmits at an interference-free rate as if undisturbed by the cognitive user. Second, the optimal parameter $\tau$ and power allocations provide useful guidance for the practical design and implementations.

\section{A. Corner Point Analysis}

As an initial analysis, we focus on special cases and derive the conditions, under which, we can solve analytically. Although these cases do not solve the problem completely, they are important bases for the more general cases and can provide intuitions and guidance. These special cases are:

1) Strong interference: any cross link is strong:

Corollary 2. When $a=0, b$ is strong $\left(b^{2} \geq\left(1+P_{1}\right)\right)$, or $b=0, a$ is strong $\left(a^{2} \geq\left(1+P_{2}\right)\right)$ or both $a$ and $b$ are strong, $R_{2}$ can achieve the maximum rate of $C\left(P_{2}\right)$ while given the condition $R_{1}=C\left(P_{1}\right)$.

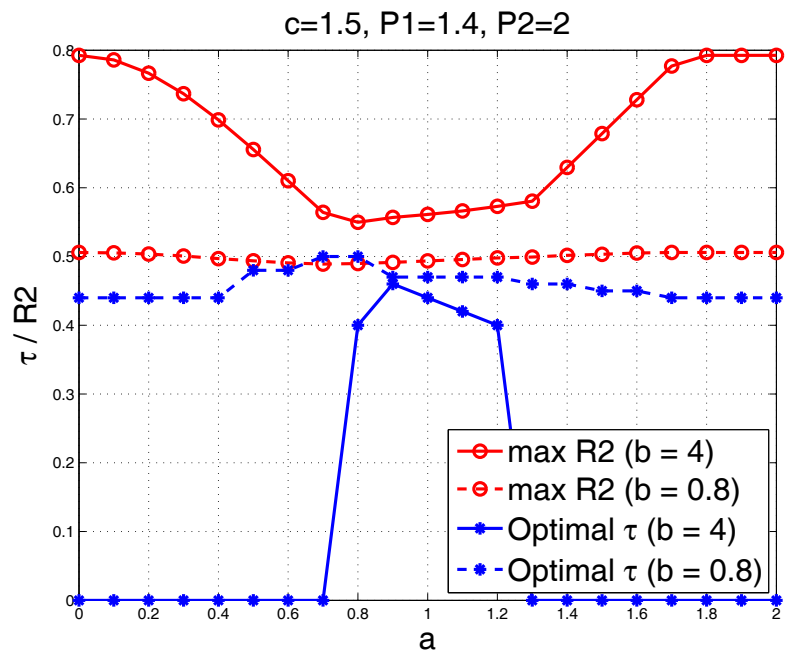

Fig. 5. Effect of a.

All 3 cases above do not require cooperation among the transmitter and the channel falls back to the classical interference channel under strong interference. The optimal parameter setting and maximum rates can be easily inferred from there.

2) Weak interference corner case: $b$ is zero, a is weak:

Corollary 3. $R_{2}$ can achieve the maximum rate of $C\left(\frac{P_{2}}{a^{2} P_{1}+1}\right)$ while $R_{1}=C\left(P_{1}\right)$ if $b=0, a$ is weak $(a<1)$.

Since $b=0$, there is no interference from cognitive to primary user, the maximum $R_{2}$ is achieved when we set

$\tau=0, \alpha_{1}=\alpha_{2}=\beta_{2}=0, \gamma_{2}=\sqrt{P_{1}}, \rho=0, \theta=\sqrt{P_{2}}, \mu=0$.

Another weak interference corner case is when $a$ is zero, and $b$ is weak. This corner case results in a complicated nonlinear optimization problem for which closed-form analytical solution does not appear feasible.

\section{B. Effect of $a, b$ and $c$}

We now numerically investigate the effect of the parameters $a, b$ and $c$ separately, and plot the relationships between the maximum $R_{2}$ and optimal $\tau$ given that $R_{1}=C\left(P_{1}\right)$.

1) Effect of $a$ : In Figure 5, we show the relationship among the maximum $R_{2}$, optimal $\tau$ and $a$ while fixing other variables. Some comments are of interest. First, when $a$ is very large, the maximum $R_{2}$ is the same as that of $a=0$. This means when the interference is strong enough, it is equivalent to having no interferences, no matter whether $b$ is strong or weak. This is valid since, when $a$ is large, $D_{2}$ receives more useful information than noise from $S_{1}$, and $D_{2}$ can decode all the information from $S_{1}$. Second, when $b$ is very strong, the maximum $R_{2}$ can achieve $C\left(P_{2}\right)$, which is the maximum possible value for $R_{2}$, either if $a$ is 0 or $a$ is very large. This also verifies the conclusion in Corollary 2. Third, when $b$ is strong, the optimal $\tau$ to achieve $C\left(P_{2}\right)$ is 0 , meaning we only have the second phase. However, when $b$ is weak, the optimal $\tau$ is nonzero. This is possible since, if $b$ is strong enough, what $S_{2}$ decodes from $S_{1}$ in the second phase is enough for forwarding. However, if $b$ is small, the lack of forwarding 


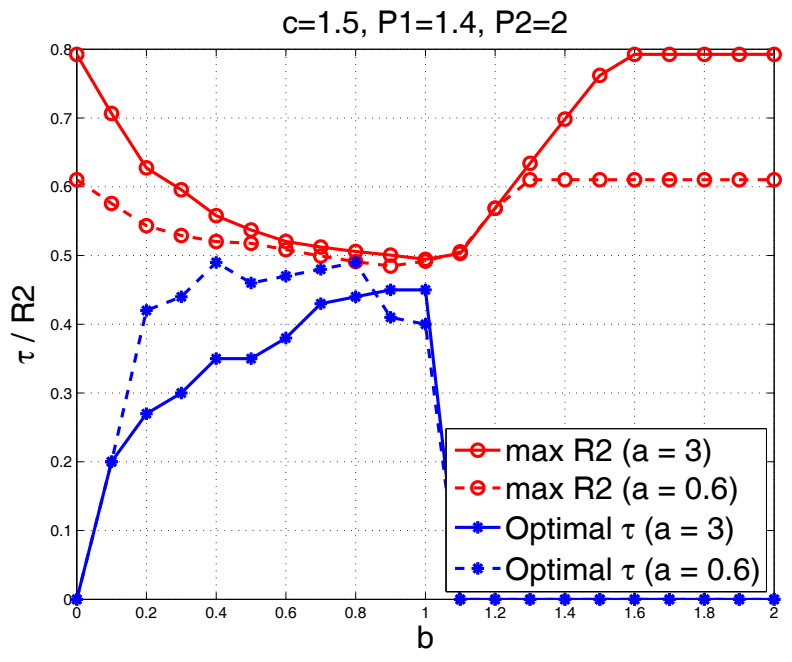

Fig. 6. Effect of b.

messages in $S_{2}$ will create a bottleneck. Thus, $S_{2}$ needs an extra phase to obtain the forwarding messages.

2) Effect of b: In Figure 6, we show the relationships between the maximum $R_{2}$, optimal $\tau$ and $b$ under the same constraint as before. We also give two different cases when $a$ is either weak or strong. Figure 6 provides supports for Corollary 2 and Corollary 3. For example, when $b=0$ and $a$ is weak (see $\max R_{2}\left(a=0.6\right.$ )), and the maximum rate for $R_{2}$ is $0.6101 \mathrm{bps} / \mathrm{Hz}$, which is exactly $C\left(\frac{P_{2}}{a^{2} P_{1}+1}\right)$. Similarly for Corollary 2 (see $\max R_{2}(a=3)$ ). Furthermore, the maximum rate for $R_{2}$ is the same either $a=0$ or $a$ is large.

3) Effect of c: In Figure 7, we show the relationship among the maximum $R_{2}$, the optimal $\tau$ and $c$. Here we only give the case when $a$ and $b$ are both weak, since when they are both strong, the cooperation via link $c$ is not necessary (see capacity for strong interference channels [8]). The value for $c$ starts from 1.0 because the cooperative link between transmitters should be no worse than the direct links; otherwise, there is no incentive for the cooperation between transmitters. The simulation result for the parameter $c$ is different from that of $a$ or $b$. First, when $c$ increases, the maximum $R_{2}$ always increases, and thus the maximum $R_{2}$ differs when $c=0$ or $c$ is strong. This makes sense because $c$ does not introduce any interference to the transmission, thus for the value of $c$, the larger the better. Second, there is a jump for $\tau$ when $c$ increases from 1.0 to 1.1. This jump is valid since when $c=1.0$, the channel between the two transmitters is no better than the direct link. Since the cross link $b<1$, there is no need for $S_{2}$ to forward the messages, thus $\tau=0$. When $c$ is above 1.0, the channel between the two senders gets better and $S_{2}$ can assist $S_{1}$ to transmit. Furthermore, as $c$ increases, the optimal $\tau$ decreases since a stronger cooperation link requires less time for user two to decode the message from user one, leaving more time for the forwarding this message.

\section{CONCLUSIONS}

In this paper, we have proposed a new transmission scheme for the half-duplex causal cognitive interference channel and

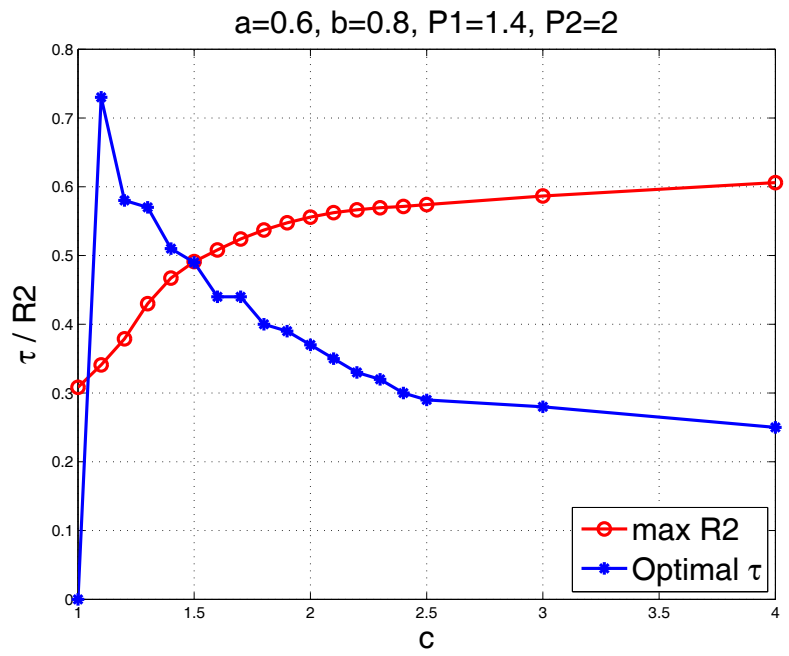

Fig. 7. Effect of c.

derive the rate region for the Gaussian case. Our considered binning scheme introduces a correlation between the transmit signal and the state, which enlarges the rate region compared with the traditional binning in dirty paper coding. Numerical results show that this scheme achieves a higher rate than the Han-Kobayashi scheme and two other existing schemes. The rate region analysis for special channel settings indicate that the cognitive user can achieve non-trivial rates while the primary user still transmits at its interference-free rate. The simulation results for different channel gains also verify this analysis. These analysis and results show that realistic overlay cognitive communications in the causal case is also beneficial.

\section{ACKNOWLEDGMENT}

The authors would like to thank Ahmad Abu Al Haija for his help in obtaining Figure 4.

\section{REFERENCES}

[1] N. Devroye, P. Mitran, and V. Tarokh, "Achievable rates in cognitive radio channels," IEEE Trans. on Information Theory, vol. 52, no. 5, pp. 1813-1827, May 2006.

[2] D. Chatterjee, T. Wong, and O. Oyman, "On achievable rate regions for half-duplex causal cognitive radio channels," in Proc. IEEE Int'l Symp. on Information Theory (ISIT), June 2010, pp. 435-439.

[3] S. Kotagiri and J. Laneman, "Multiaccess channels with state known to some encoders and independent messages," EURASIP J. Wireless Comm. and Networking Theory and App. in Multiuser/Multiterminal Comm., vol. 2008, no. 24, pp. 1-14, Jan. 2008.

[4] M. Costa, "Writing on dirty paper (corresp.)," IEEE Trans. on Information Theory, vol. 29, no. 3, pp. 439-441, May 1983.

[5] A. Carleial, "Interference channels," IEEE Trans. on Information Theory, vol. 24, no. 1, pp. 60-70, Jan. 1978.

[6] Z. Wu and M. Vu, "Partial decode-forward binning for full-duplex causal cognitive interference channels," in Proc. IEEE Int'l Symposium on Information Theory, July 2012, pp. 1331-1335. (Longer version under the title "Partial decode-forward binning schemes for the causal cognitive relay channels" is available at http://arxiv.org/abs/1111.3966).

[7] A. Host-Madsen and J. Zhang, "Capacity bounds and power allocation for wireless relay channels," IEEE Trans. on Information Theory, vol. 51, no. 6, pp. 2020-2040, June 2005.

[8] H. Sato, "The capacity of the gaussian interference channel under strong interference (corresp.)," IEEE Trans. on Information Theory, vol. 27, no. 6, pp. 786-788, Nov. 1981. 